\title{
A Causal Set Black Hole
}

\author{
Song $\mathrm{He}^{*}$ \\ School of Physics, Peking University, Beijing, 100871, China, \\ David Rideout ${ }^{\dagger}$ \\ Perimeter Institute for Theoretical Physics, \\ 31 Caroline St. N, Waterloo, Ontario N2L 2Y5, Canada
}

October 30, 2018

\begin{abstract}
We explicitly compute the causal structure of the Schwarzschild black hole spacetime, by providing an algorithm to decide if any pair of events is causally related. The primary motivation for this study comes from discrete quantum gravity, in particular the causal set approach, in which the fundamental variables can be thought of as the causal ordering of randomly selected events in spacetime. This work opens the way to simulating non-conformally flat spacetimes within the causal set approach, which may allow one to study important questions such as black hole entropy and Hawking radiation on a full four dimensional causal set black hole.
\end{abstract}

\section{Contents}

1 Introduction

2 Null geodesics and the causal structure of a Schwarzschild black hole 3

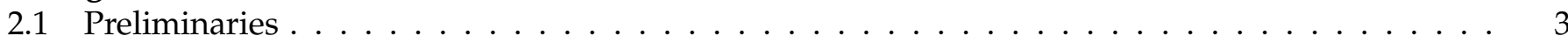

2.2 Radially separated pairs and radial null geodesics . . . . . . . . . . . . . . . . 5

2.3 Sufficient conditions for causally related and unrelated pairs . . . . . . . . . . . . . .

2.3 .1 Spacelike bounds . . . . . . . . . . . . . . . . . . . . . . . 7

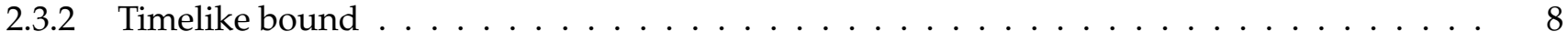

2.4 Generic pairs of events and null geodesics . . . . . . . . . . . . . . . . . . 9

3 Results $\quad 10$

3.1 Causal Relations . . . . . . . . . . . . . . . . . . . . . . . . 10

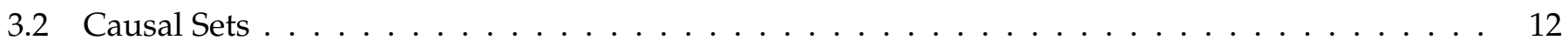

$\begin{array}{lll}4 & \text { Conclusion and discussion } & 17\end{array}$

\begin{tabular}{ll} 
A Appendix: Proof of the proposition & 18 \\
\hline
\end{tabular}

*Email address: hesong@pku.edu.cn

${ }^{\dagger}$ Email address: drideout@perimeterinstitute.ca 
B.1 Sprinkling into Schwarzschild Spacetime . . . . . . . . . . . . . . . . . . . . 18

B.2 Determining the Causal Relations . . . . . . . . . . . . . . . . . . . . . . 19

\section{Introduction}

Much is understood about the causal structure of the Schwarzschild black hole spacetime, e.g. in the sense that the collection of all null geodesics has been completely classified [1]. However, what is missing is a complete specification of the causal relations, namely the set of ordered pairs of events in Schwarzschild which are connected by a causal curve. It is the purpose of this paper to provide exactly such a prescription: Given an arbitrary ordered pair of events in Schwarzschild spacetime, does there exist a future directed causal curve from the first to the second?

Unfortunately, the differential equations describing the null geodesics are solvable in closed form only for limited values of their parameters [1]. (In fact only a set of measure zero are expressible in closed form.) We therefore describe an algorithm which will allow one to compute an answer to the above question, to any (finite) precision. For more implicit discussion on how the space of null geodesics encodes the causal structure of Schwarzschild, and general spacetimes, please refer to [2, 3] and references therein.

The primary motivation for this work comes from discrete quantum gravity, in particular the causal set approach, for which the fundamental variables can be regarded as the causal ordering of randomly selected events in spacetime [4, 5, 6]. More specifically the causal set is a discrete set of 'atoms of spacetime', which possesses a partial order relation which corresponds to causal ordering in spacetime. Because of this straightforward interpretation of the fundamental variables, it is relatively easy to extract phenomenological predictions from the theory on the effects of fundamental spacetime discreteness. Perhaps the most famous of these is the prediction of a small but non-zero fluctuating cosmological constant, whose current order of magnitude matches its observed value [7].

Mathematically a causal set is a set $C$ endowed with an order relation $\prec$ with is irreflexive $(x \nprec x)$, transitive ( $x \prec y$ and $y \prec z \Longrightarrow x \prec z$ ), and locally finite $(|\{y \mid x \prec y \prec z\}|$ is finite for all $x, z \in C) \sqrt{1}$. The connection between a microscopic discrete causal set and a macroscopic approximating continuum spacetime arises via the notion of a 'sprinkling', which is a simple algorithm for generating a causal set from a spacetime. Given a spacetime $M$ with finite spacetime volume $V$ (such as a bounded region of an infinite spacetime), select at random $N$ events in $M$, with respect to the volume measure. By this we mean that in any region of $M$ of volume $v$ one expects to find $n$ sprinkled events, where $n$ is sampled from a Poisson distribution of mean $v$. Thus the probability of finding $n$ sprinkled events in a region of volume $v$ is

$$
\operatorname{Pr}(n, v)=\frac{v^{n} e^{-v}}{n !}
$$

(Therefore $N$ is sampled from the distribution Eq. (1) with $v=V$.) Each of these sprinkled events then corresponds to an element of the causal set. After sprinkling the $N$ events, one defines the partial ordering by stating that two elements are related iff the events are causally related in the spacetime. The microscopic-continuum correspondence then arises by the statement that if a causal set is likely to have arisen from a sprinkling into a given spacetime, then one regards that spacetime as a good macroscopic approximation of the underlying causal set.

Thus far only conformally flat spacetimes, viz. those whose metric is given by a scalar conformal times the Minkowski metric, have been used in detailed calculations involving sprinklings of causal sets, because the second step of deducing the causal relations is particularly easy in that case. Here we open the possibility for sprinkling into a non-conformally flat spacetime: the Schwarzschild black hole. This allows one to address the general question of whether the methods of deducing properties of continuum spacetime from the causal set

\footnotetext{
${ }^{1}$ The vertical brackets $|\cdot|$ stand for cardinality.
} 
carry over to curved spacetime, and in particular spacetime with non-flat conformal structure. Some constructs which one may like to test are dimension estimators [5, 8], the recovery of lengths of timelike [6, 9] and spacelike [10] geodesics, and extraction of macroscopic spatial topology [11].

The black hole is important for a number of other reasons, beyond merely being an example of a nonconformally flat spacetime. In the same way in which one can get a first approximation of the entropy of a gas merely by counting molecules, there is now substantial evidence that one can do an analogous counting of 'horizon molecules', such as causal links of a causal set crossing the horizon, to compute the entanglement entropy of a black hole, and thus get a handle on its physical origin [12]. In addition one may test a recently proposed entropy bound [13] in this spherically symmetric, yet conformally curved context. Finally, this work can shed light on the longstanding problem of the role trans-Planckian modes in Hawking radiation, by allowing the study of a full four dimensional causal set black hole.

The technique we present here, of deducing the causal relation from a classification of the null geodesics of a spacetime, can be generalized to other spacetimes as well. The most obvious example is the ReissnerNordstrom black hole. It remains spherically symmetric, which considerably simplifies the analysis, and its null geodesics are completely classified [1]. Presumably one could also analyze the Kerr black hole in this way, though the broken spherical symmetry will be considerably more complicated. Note also that one must avoid any regions which contain closed timelike curves (or consider a more general ordered substructure), since the causal set description breaks down there.

In addition to describing an algorithm to decide if any two events in Schwarzschild are connected by a causal curve, we implement this prescription as a 'thorn' (module) in the Cactus high performance computing framework [14]. An advantage of doing so is that others can easily make use of the code, without having to write their own implementation of the algorithm, nor having to understand the details of the representation of the causal set on the computer.

This paper is organized as follows. In section 2 we briefly describe the equations governing the null geodesics we will employ, and describe how to use them to determine if two events are causally related or not. In section 3 we present some of the causal relations as computed by our algorithm, and pictures which illustrate the causal sets which arise by sprinkling into a region of Schwarzschild spacetime. Section 4 contains some concluding remarks. In Appendix $\mathrm{A}$ we give a proof that the null geodesics we integrate to determine the causal relations are those that arrive earliest. In Appendix $B$ we describe the details of our implementation of this method, including how to sprinkle into the Schwarzschild geometry with uniform density.

\section{Null geodesics and the causal structure of a Schwarzschild black hole}

\subsection{Preliminaries}

In this paper, we want to find a general recipe to determine unambiguously whether two events in four dimensional Schwarzschild spacetime are causally related to each other. This problem has a simple answer in Minkowski spacetime, since it is straightforward to show that if $-\left(t_{2}-t_{1}\right)^{2}+\left(x_{1}-x_{2}\right)^{2}+\left(y_{1}-y_{2}\right)^{2}+\left(z_{1}-z_{2}\right)^{2} \leq 0$, two events $E_{1}=\left(t_{1}, x_{1}, y_{1}, z_{1}\right)$ and $E_{2}=\left(t_{2}, x_{2}, y_{2}, z_{2}\right)$ are causally related, otherwise there is no causal relation between them. Things become more complicated in curved spacetime, where in principle we need to integrate the infinitesimal invariant distance $d s$ along every possible path from one event to the other to see if there is a null or timelike curve (causal curve) connecting them.

Nevertheless, it is possible to solve this problem, given an understanding of the collection of null geodesics in the spacetime. In the Schwarzchild metric, it is always possible to choose a time parameter that only increases towards future, i.e. $\partial_{t}^{a}$ is everywhere a future pointing timelike vector 2 Then given two events $E_{1}$ and $E_{2}$ with

\footnotetext{
${ }^{2}$ We will choose Eddington-Finkelstein coordinates, which have a such a time parameter, below.
} 


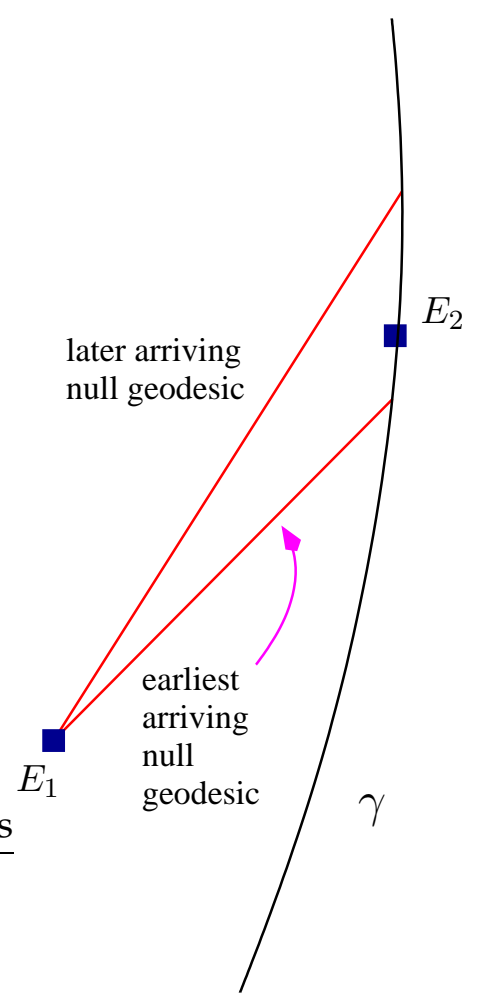

Figure 1: Illustration of the timelike worldline $\gamma$, on which all spatial coordinates (including $r$ ) are constant, and two null geodesics from $E_{1}$ to $\gamma$.

$t_{1}<t_{2}$, the only possible causal relation between them is that $E_{1} \prec E_{2}$, which means that there is a futuredirected causal curve from $E_{1}$ to $E_{2}$. Imagine a bunch of light rays (null geodesics) $B$, emanating from $E_{1}$, and $E_{2}$ represents a particular moment $t_{2}$ in the world line $\gamma$ of a stationary observer. If the world line of any null geodesics in $B$, which is a null geodesic, meets $\gamma$ at $t \leq t_{2}$, then we can conclude that they are causally-related. On the other hand, if any null geodesic emanating from $E_{1}$ can only reach $\gamma$ at $t>t_{2}$, then $E_{1}$ and $E_{2}$ must be causally unrelated to each other. See Fig. 1 for an illustration. That this is true can be seen by proposition 2.20 of [15], which states if $A \in J^{-}(B)$ but $A \notin I^{-}(B)$, then there must exist a future-directed null geodesic from $A$ to $B$. This tells us that the earliest future-directed causal curve must be a null geodesic, but since any null geodesic has failed to reach $\gamma$ early enough for a causal relation between the two events, then the conclusion is that there is no causal relation between them.

We will prove in Appendix $\mathrm{A}$ that our procedure always considers the fastes $3^{3}$ geodesic from $E_{1}$ to $\gamma$, so the arrival time $t$ at $\gamma$ of that geodesic will be sufficient to determine if $E_{1}$ and $E_{2}$ are causally related. If we find that even this null geodesic meets $\gamma$ later than $t_{2}$, we can say for sure that there is no way for these two events to be connected by any future directed causal curve.

After this brief introduction, we discuss in the next subsection the particular simple case where $E_{1}$ and $E_{2}$ are only radially separated, with no angular separation, so all we need to consider are radial geodesics. For more generic pairs of events, we must consider the full three dimensional case 4 which is too complicated for a complete analytic solution. However, before incorporating a numerical treatment for the generic case, in subsection 2.3 we find two simple sufficient conditions for two events to be causally unrelated to each other. One uses a bound given by radial null geodesics, and the other by purely angular components. Furthermore, there is also a sufficient condition for two events to have causal relation, which is the existence of a composed null

\footnotetext{
${ }^{3} \mathrm{By}^{\prime}$ 'fastest' we mean the geodesic with the earliest arrival time, as given by the (EF) time coordinate.

${ }^{4}$ Given two events in Schwarzschild spacetime, is is always possible to rotate the coordinates so that they both lie in the equatorial plane, as explained below.
} 
curve connecting $E_{1}$ and an event in $\gamma$ no later than $E_{2}$. These sufficient conditions are enough to determine the causal relations for a large portion of pairs of events in Schwarzschild spacetime, and provide a very efficient preconditioning, since we only need to do numerical calculations for those which fail all these conditions. Furthermore, we shall give the recipe for generic pairs of events in subsection 2.4, with the help of numerical calculations. As mentioned above, the proof of the lemma which ensures that the null geodesic found by our recipe is the fastest one connecting $E_{1}$ and $\gamma$ is given in the Appendix.

We start with the Schwarzschild metric in the familiar form

$$
d s^{2}=-\left(1-\frac{2 M}{r}\right) d t_{s}^{2}+\left(1-\frac{2 M}{r}\right)^{-1} d r^{2}+r^{2}\left(d \theta^{2}+\sin ^{2} \theta d \phi^{2}\right),
$$

where $M$ is the mass of the black hole. The metric is well known to possess a coordinate singularity at $r=2 M$, where the event horizon lies. It can be written in Eddington-Finkelstein (EF) coordinates [16],

$$
d s^{2}=-d t^{2}+d r^{2}+r^{2}\left(d \theta^{2}+\sin ^{2} \theta d \phi^{2}\right)+\frac{2 M}{r}(d t+d r)^{2},
$$

with the following transformation of time parameter,

$$
t=t_{s}+2 M \ln \left(\frac{r}{2 M}-1\right) .
$$

Given two events in the EF coordinates, $E_{1}=\left(t_{1}, r_{1}, \theta_{1}, \phi_{1}\right)$ and $E_{2}=\left(t_{2}, r_{2}, \theta_{2}, \phi_{2}\right)$ with $t_{1} \leq t_{2}$, the only possible causal relation is $E_{1} \prec E_{2}$. Besides, it is obvious that one can always choose suitable angular coordinates $\vartheta$ and $\varphi$ for which $\vartheta_{1}=\vartheta_{2}=\pi / 2, \varphi_{1}=0$, and $\varphi_{2}=\arccos \left(\cos \theta_{1} \cos \theta_{2}+\sin \theta_{1} \sin \theta_{2} \cos \left(\phi_{1}-\phi_{2}\right)\right) \in$ $[0, \pi]$. Therefore, it is sufficient to consider a pair of events $E_{1}=\left(t_{1}, r_{1}, \pi / 2,0\right)$ and $E_{2}=\left(t_{2}, r_{2}, \pi / 2, \varphi_{2} \in[0, \pi]\right)$ with $t_{1} \leq t_{2}$. The stationary worldline $\gamma$ containing $E_{2}$ is given by $\left(t, r=r_{2}, \vartheta=\frac{\pi}{2}, \varphi=\varphi_{2}\right)$, i.e. all spatial coordinates are held fixed. We define the angle through which our null geodesic travels, $\Delta \varphi$, by

$$
\Delta \varphi=\int_{\eta} d \varphi
$$

where $\eta$ is a null geodesic from $E_{1}$ to $\gamma$, and $\varphi$ is the azimuthal coordinate in the rotated coordinate system. In subsection 2.4 we shall find that a generic null geodesic from $E_{1}$ to $\gamma$ can always be formulated as one with $\vartheta=\pi / 2$ as well, thus the whole problem, including the events and geodesics in between, can be projected to $2+1$ dimensions.

An important question when considering the generic pair of events will be if the null geodesic we choose is the fastest one, i.e. that it arrives at $\gamma$ before all other null geodesics from $E_{1}$. In order for the null geodesic $\eta$ to arrive at $\gamma$, it must have $\Delta \varphi=2 k \pi+\varphi_{2}$, with $k \in \mathbb{Z}$. In Appendix A we prove that a geodesic $\eta$ with $|\Delta \varphi|=\varphi_{2}$ is a fastest future directed null geodesic from $E_{1}$ to $\gamma 5$ Thus we only need to consider those null geodesics from $E_{1}$ to $\gamma$ which travel for an angle no more than $\pi$ in the $\varphi$ direction.

\subsection{Radially separated pairs and radial null geodesics}

Now let us consider the simplest case with $\varphi_{2}=0$. In this case we only need to consider radial null geodesics, and it is straightforward that in the EF coordinates, by setting $d \vartheta=d \varphi=0$ and $d s^{2}=0$, radial null geodesics take a simple form,

$$
\begin{gathered}
d t+d r=0 \\
\left(\frac{2 M}{r}-1\right) d t+\left(\frac{2 M}{r}+1\right) d r=0
\end{gathered}
$$

\footnotetext{
${ }^{5}$ The absolute value on $|\Delta \varphi|$ is merely to account for the case of $\varphi_{2}=\pi$, in which there are two fastest null geodesics, i.e. that both have the same arrival time. However, note that this case will never arise in the sprinkling described in Section 1 as the set of such sprinklings is of measure zero in the space of all sprinklings.
} 
where the first one describes ingoing null geodesics, while the second one turns out to be outgoing for null geodesics outside the event horizon, and ingoing for those inside the event horizon. The two radial directions for null geodesics, both outside and inside the horizon, are illustrated in Fig. 2. Therefore, given two events

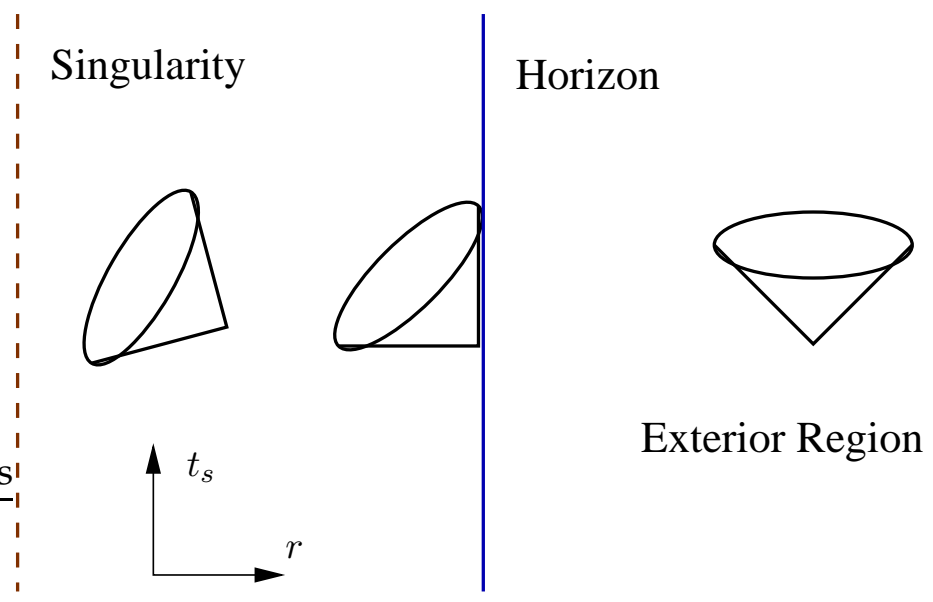

Figure 2: Schematic illustration of lightcones in a Schwarzschild black hole. The event horizon is indicated by the blue line, and the interior region is to the left. Note the two ingoing radial directions for future directed lightcones in the interior region. (The vertical direction is to be regarded here as Schwarzschild time, which can go backward for timelike observers inside the horizon.)

without angular separation, $E_{1}=\left(t_{1}, r_{1}, \pi / 2,0\right)$ and $E_{2}=\left(t_{2}, r_{2}, \pi / 2,0\right)$ with $t_{1} \leq t_{2}$, we can determine if they are causally related by considering the time for null geodesics from $E_{1}$ to reach a point in $\gamma$, the world line of a stationary observer at $\left(r_{2}, \pi / 2,0\right)$.

First assume $r_{1} \geq r_{2}$. We consider only ingoing null geodesics from $r_{1}$ to $r_{2}$. If both events are outside the horizon, $r_{1} \geq r_{2}>2 M$, there are only ingoing null geodesics given by Eq. (4), and it is straightforward to see that these two events are causally related to each other if and only if $t_{2} \geq t_{1}+r_{1}-r_{2}$, where $t_{1}+r_{1}-r_{2}$ is simply the time when a null geodesic from $E_{1}$ hits $\gamma$. If $r_{1} \geq 2 M \geq r_{2}$, we still only have Eq. (4) for ingoing rays outside the horizon, and to ensure continuity of the tangent of the geodesic when it crosses the horizon, we can only use Eq. (4) inside the horizon too, and the conclusion is not altered. However, if both events are inside the horizon, $2 M \geq r_{1} \geq r_{2}$, we have two choices of ingoing rays given by Eq. (4) and Eq. (5), and a simple calculation shows that it takes a shorter time for a null geodesic from $E_{1}$ to hit $\gamma$ for the first case, $d t=-d r=|d r|$, than for the second case $d t=-\frac{2 M / r+1}{2 M / r-1} d r=\left(1+\frac{2 r}{2 M-r}\right)|d r|>|d r|$ for $r<2 M$. Therefore, for both events inside the horizon, the necessary and sufficient condition for their causal relation is still $t_{2} \geq t_{1}+r_{1}-r_{2}$.

On the other hand, if $r_{2} \geq r_{1}$, we shall instead consider outgoing rays, given by Eq. (5) outside the horizon. There is a special case, $r_{1}<2 M$, for which no matter $r_{2} \leq 2 M$ or $r_{2}>2 M$, they can not be causally related to each other for any $t_{1}$ and $t_{2}$, since there are no outgoing null geodesics either propagating inside the horizon or coming out of it. If they both lie outside the horizon, $r_{2} \geq r_{1}>2 M$, then the sufficient and necessary condition for a causal relation between them is $t_{2} \geq t_{1}+r_{2}-r_{1}+4 M \ln \left(\frac{r_{2}-2 M}{r_{1}-2 M}\right)$, where $t_{1}+r_{2}-r_{1}+4 M \ln \left(\frac{r_{2}-2 M}{r_{1}-2 M}\right)=$ $t_{1}+\int_{r_{1}}^{r_{2}} \frac{r+2 M}{r-2 M} d r$ is the time for a null geodesic given by Eq. (5) to travel from $E_{1}$ to the point it meets $\gamma$.

To summarize, we have the following recipe to determine if two events are causally related to each other. If $r_{1} \geq r_{2}$, the (necessary and sufficient) condition for a causal relation is always

$$
t_{2} \geq t_{1}+r_{1}-r_{2},
$$

while if $r_{2} \geq r_{1}>2 M$, they are related if and only if

$$
t_{2} \geq t_{1}+r_{2}-r_{1}+4 M \ln \left(\frac{r_{2}-2 M}{r_{1}-2 M}\right) .
$$

Finally if $r_{2}>r_{1}$ and $r_{1}<2 M$, they can not be causally related to each other. 


\subsection{Sufficient conditions for causally related and unrelated pairs}

Since in practice we are interested in computing the causal relations between many pairs of events, it is useful to derive bounds that allow one to decide whether the events are related quickly, based on simple criteria, without having to perform any numerical integrations. In this section we derive such bounds, which are sufficient to determine that the two events are unrelated (section 2.3.1) or related (section 2.3.2).

\subsubsection{Spacelike bounds}

For two events with non-zero angular distance, i.e. $\varphi_{2} \in(0, \pi]$, we have to consider non-radial, three dimensional null geodesics in general [1]. Nevertheless, we shall find two independent sufficient conditions for the events to be causally unrelated below, by using lower bounds on the time duration of any future-directed causal curve from $E_{1}$ to $\gamma$, given by either radial null geodesics with the angular part discarded, or by a purely angular component with the radial part discarded.

Since anywhere on a future-directed causal curve we have $d s^{2} \leq 0$ and $d t>0$, with Eq. (2) we obtain an inequality by discarding the (positive) angular part,

$$
(d t+d r)\left[\left(\frac{2 M}{r}-1\right) d t+\left(\frac{2 M}{r}+1\right) d r\right] \leq 0
$$

If $r>2 M$, we obtain for an ingoing causal curve $d r<0, d t \geq-d r$, and for an outgoing curve $d r>0$, $d t \geq \frac{r+2 M}{r-2 M} d r$. If $r<2 M$, we obtain for an ingoing curve $d r<0, \frac{r+2 M}{r-2 M} d r \geq d t \geq-d r$, and no solution for an outgoing curve $d r>0$.

These inequalities can be integrated along the whole curve from $E_{1}$ to $\gamma$ to give a lower bound on the time duration, which turns out to be exactly the time duration of radial null geodesics from $r_{1}$ to $r_{2}$. If $r_{1} \geq r_{2}$, the sufficient condition for two events to be causally unrelated is

$$
t_{2}-t_{1}<r_{1}-r_{2}
$$

while if $r_{2} \geq r_{1}>2 M$, they are unrelated if

$$
t_{2}-t_{1}<r_{2}-r_{1}+4 M \ln \left(\frac{r_{2}-2 M}{r_{1}-2 M}\right) .
$$

Finally if $r_{2}>r_{1}$ and $r_{1}<2 M$, they can not be causally related to each other.

On the other hand, we can also try to discard the radial part to obtain a bound by using a purely angular component, but this is more subtle and we need to be careful. Since $d r^{2}+\frac{2 M}{r}(d t+d r)^{2} \geq 0$, we can discard this part to obtain an inequality anywhere on a causal curve, as $-d t^{2}+r^{2} d \varphi^{2} \leq 0$, which gives $d t \geq r|d \varphi|$. However, for outgoing causal curves outside the horizon, we have $\left(1+\frac{2 M}{r}\right) d r^{2}+\frac{\overline{4} M}{r} d t d r>0$ and a stronger bound can be obtained as $\left(\frac{2 M}{r}-1\right) d t^{2}+r^{2} d \varphi^{2} \leq 0$ which gives $d t \geq \frac{r|d \varphi|}{\sqrt{1-2 M / r}}$, where $r>2 M$. The equality holds for null geodesics with constant $r$, which satisfy

$$
\sqrt{1-\frac{2 M}{r}} d t \pm r d \varphi=0
$$

where $r=$ const $>2 M$. This can be rephrased physically by stating that it is impossible for a particle inside the horizon to move around an orbit with constant $r<2 M$, because nothing can stop it from falling into the singularity $r=0$. We shall make direct use of Eq. (8) later for the timelike bound.

For any ingoing curve from $E_{1}$ to $\gamma$, we have $d t \geq r|d \varphi| \geq r_{2}|d \varphi|$ along it, and we obtain a sufficient condition for two events with $r_{1} \geq r_{2}$ to be causally unrelated,

$$
t_{2}<t_{1}+r_{2} \varphi_{2}
$$


For any outgoing curves from $E_{1}$ to $\gamma$, we have $d t \geq \frac{r|d \varphi|}{\sqrt{1-2 M / r}}$ along it, and we need to find out the minimum of $f(r)=\frac{r}{\sqrt{1-2 M / r}}$ in the range $2 M<r_{1} \leq r \leq r_{2}$. It is straightforward to obtain

$$
f^{\prime}(r)=\frac{1-3 M / r}{(1-2 M / r)^{3 / 2}},
$$

from which the location of the minimum can be determined to be $r_{0}=r_{1}$ for $3 M \leq r_{1} \leq r_{2}, r_{0}=3 M$ for $r_{1}<3 M<r_{2}$, and $r_{0}=r_{2}$ for $r_{1} \leq r_{2} \leq 3 M$.

Therefore we have $d t \geq \frac{r_{0}|d \varphi|}{\sqrt{1-2 M / r_{0}}}$ along the curve and we obtain a sufficient condition for two events with $2 M<r_{1} \leq r_{2}$ to be causally unrelated,

$$
t_{2}-t_{1}<f\left(r_{0}\right) \varphi_{2}
$$

\subsubsection{Timelike bound}

Furthermore, it turns out that for many pairs, as long as at least one of them is outside the horizon, we can use radial null geodesics and null geodesics with constant $r$ to find a composed null curve connecting $E_{1}$ and an event in $\gamma$. If this event is no later than $E_{2}$, then this will be a sufficient condition for their causal relation.

Given Eq. (4), Eq. (5) and Eq. (8), we can construct a null curve from $E_{1}$ to an event in $\gamma$ whenever $r_{1}>2 M$, which is composed of a sequence of null geodesics with constant $\varphi$ and constant $r$. To optimize this sufficient condition for a causal relation, we need to minimize the time duration of the segment of the null geodesic with constant $r$, which occurs at $r=r_{0}$ where $r_{0}=\min \left(r_{1}, r_{2}\right)$ for $r_{1}, r_{2} \geq 3 M, r_{0}=3 M$ for $r_{1}>3 M>r_{2}$ or $r_{2}>3 M>r_{1}$, and $r_{0}=\max \left(r_{1}, r_{2}\right)$ for $r_{1}, r_{2} \leq 3 M$.

If $r_{1} \geq r_{0} \geq r_{2}$, then we can compose the null curve by the following three segments:

1. an ingoing radial segment from $r=r_{1}$ to $r=r_{0}$ with $\varphi=0$;

2. a segment from $\varphi=0$ to $\varphi=\varphi_{2}$ with $r=r_{0}$;

3. an ingoing radial segment from $r=r_{0}$ to $r=r_{2}$ with $\varphi=\varphi_{2}$.

The time for this null curve to reach $\gamma$ is easy to compute by Eq. (4) and Eq. (8),

$$
t=t_{1}+r_{1}-r_{2}+f\left(r_{0}\right) \varphi_{2} .
$$

Here we do not care about whether $r_{2}$ is inside or outside the horizon, since regardless of whether the segment 3 lies completely outside the horizon or crosses the horizon, we always use Eq. (4) for continuity. Similarly, if $r_{2} \geq r_{0} \geq r_{1}>2 M$, then we can construct the null curve by replacing segments 1 and 3 above by outgoing radial segments, and the time can be computed by Eq. (5) and Eq. (8) to be

$$
t=t_{1}+r_{2}-r_{1}+4 M \ln \left(\frac{r_{2}-2 M}{r_{1}-2 M}\right)+f\left(r_{0}\right) \varphi_{2} .
$$

Therefore, we have the following sufficient condition for two events, of which at least one is outside the horizon, to be causally related. If $r_{1} \geq r_{2}$ and $r_{1}>2 M$, then they are causally related if

$$
t_{2} \geq t_{1}+r_{1}-r_{2}+f\left(r_{0}\right) \varphi_{2}
$$

if $r_{2} \geq r_{1}>2 M$, they are causally related if

$$
t_{2} \geq t_{1}+r_{2}-r_{1}+4 M \ln \left(\frac{r_{2}-2 M}{r_{1}-2 M}\right)+f\left(r_{0}\right) \varphi_{2} .
$$

If a pair of events fails both of these sufficient conditions, then we have to consider a generic form of null geodesics to determine if they are causally related. 


\subsection{Generic pairs of events and null geodesics}

The most generic null geodesics in Schwarzschild spacetime have the following form [1],

$$
p_{t} \frac{d t}{d \tau}-p_{r} \frac{d r}{d \tau}-p_{\theta} \frac{d \theta}{d \tau}-p_{\phi} \frac{d \phi}{d \tau}=0
$$

where $\tau$ is an affine parameter, and $p_{t}, p_{\phi}$ are constants,

$$
\begin{gathered}
p_{t}=\left(1-\frac{2 M}{r}\right) \frac{d t_{s}}{d \tau}=E, \\
p_{\phi}=r^{2} \sin ^{2} \theta \frac{d \phi}{d \tau}=L,
\end{gathered}
$$

and $p_{\phi}$ satisfies

$$
\frac{d\left(r^{2} \frac{d \theta}{d \tau}\right)}{d \tau}=r^{2} \sin \theta \cos \theta\left(\frac{d \phi}{d \tau}\right)^{2}
$$

If we choose $\vartheta=\pi / 2$ at the moment when $\frac{d \vartheta}{d \tau}=0$, we get also $\frac{d^{2} \vartheta}{d \tau^{2}}=0$ at this moment, which implies $\vartheta=\pi / 2$ all along the geodesic. Therefore a general null geodesic can be described in the plane $\vartheta=\pi / 2$, which also simplifies its equations to

$$
\left(\frac{d r}{d \tau}\right)^{2}+\frac{L^{2}}{r^{2}}\left(1-\frac{2 M}{r}\right)=E^{2},
$$

where $E$ and $L$ denote the constant energy and angular momentum of the massless particle,

$$
\begin{aligned}
\left(1-\frac{2 M}{r}\right) \frac{d t_{s}}{d \tau} & =E, \\
\frac{d \varphi}{d \tau} & =\frac{L}{r^{2}} .
\end{aligned}
$$

The energy is expressed in terms of the Schwarzschild time parameter $t_{s}$.

The full set of Eq. (11) and Eq. (12), combined with initial values of $t, r$ and $\varphi$, as well as $E$ and $L$, can uniquely determine a null geodesic in Schwarzschild spacetime. However, since we only want to obtain relations between $t, r$ and $\varphi$ without the affine parameter $\tau$, it is convenient to consider $r$ as a function of $\varphi$ and use a new variable $u=1 / r$. We then obtain from Eq. (11),

$$
\left(\frac{d u}{d \varphi}\right)^{2}=2 M u^{3}-u^{2}+c^{2}
$$

or equivalently,

$$
\frac{d \varphi}{d u}= \pm\left(2 M u^{3}-u^{2}+c^{2}\right)^{-1 / 2}
$$

where + corresponds to $d \varphi / d u>0$, - corresponds to $d \varphi / d u<0$, and $c=E / L$ for $L \neq 0$. The case of $L=0$, which corresponds to radial null geodesics, has been discussed in Subsection 2.2. It turns out that, for $L \neq 0$, the geodesic depends on $E$ and $L$ only through their ratio $c$. Using Eq. (3) and Eq. (12), we can further obtain

$$
\frac{d t}{d \varphi}=\frac{c r^{2}}{1-2 M / r}+\frac{d r / d \varphi}{r / 2 M-1}
$$

which can be simplified by using the new variable $u=1 / r$ and Eq. (13) as

$$
\frac{d t}{d \varphi}=\frac{c \mp 2 M u \sqrt{2 M u^{3}-u^{2}+c^{2}}}{u^{2}-2 M u^{3}} .
$$


Alternatively, we can put it into an equation involving only $t$ and $u$,

$$
\frac{d t}{d u}=\frac{ \pm c\left(2 M u^{3}-u^{2}+c^{2}\right)^{-1 / 2}-2 M u}{u^{2}-2 M u^{3}}
$$

where, as mentioned before, + corresponds to $d \varphi / d u>0$, - corresponds to $d \varphi / d u<0$. Eq. (13) and Eq. (14), or equivalently Eq. (13) and Eq. (15) is a full set of equations for generic null geodesics with non-zero angular momenta.

Now given $E_{1}$ and $E_{2}$ which do not satisfy any of the sufficient conditions in Subsection 2.3, we have to do the following numerical calculation to see if they are causally related to each other. For any $c$ we can integrate Eq. (13) from $\varphi_{1}=0, u_{1}=1 / r_{1}$ to $u_{2}=1 / r_{2}$, and get some value $\varphi_{2}^{\prime}$. By choosing a suitable $c_{0}$, we can make $\varphi_{2}^{\prime}=\varphi_{2}$ which means that the null geodesic with $c_{0}$ hits $\gamma$ from $E_{1}$. Then we can use $c_{0}$ in Eq. (15), and integrate it from $u_{1}, t_{1}$ to $u_{2}$ and get some value $t$. If $t \leq t_{2}$, then they are definitely causally related. If $t>t_{2}$, according to the lemma of Section 2.1, they must be causally unrelated.

\section{Results}

\subsection{Causal Relations}

We sprinkle into a region of Schwarzschild spacetime, which is bounded by $0 \leq r \leq r_{\max }$ and $0 \leq t \leq t_{\max }$. See Appendix B.1 for details on how this is done. It is important to note that in this section we use the unrotated coordinates, so $\theta$ is not restricted to $\frac{\pi}{2}$, and use coordinates such that $M=1$. By 'equatorial plane', we simply mean that $\theta=\frac{\pi}{2}$.

Nine events selected from a region of Schwarzschild with $r_{\max }=3, t_{\max }=8$, are shown in Table 16

\begin{tabular}{|c|cccc|l|}
\hline event & $t$ & $r$ & $\theta$ & $\phi$ & \\
\hline 0 & 0.410895 & 2.36161 & 1.80295 & 0.57951 & exterior \\
1 & 1.109415 & 2.89891 & 1.04335 & 4.25531 & exterior \\
2 & 1.133105 & 1.36083 & 1.89919 & 1.06482 & interior \\
3 & 2.743428 & 2.74093 & 2.97906 & 4.22204 & exterior \\
4 & 3.235970 & 0.65462 & 0.11664 & 5.06884 & interior \\
5 & 3.972871 & 0.96354 & 2.33727 & 1.38169 & interior \\
6 & 5.230757 & 2.34476 & 1.11855 & 3.47242 & exterior \\
7 & 6.014261 & 0.664739 & 2.82235 & 0.95459 & interior \\
8 & 6.193089 & 0.429636 & 2.20122 & 1.99644 & interior \\
\hline
\end{tabular}

Table 1: Nine events in Schwarzschild spacetime, specified by their Eddington-Finkelstein coordinates.

Our task is to decide, for each pair of events $\left(E_{1}, E_{2}\right)$ (with $E_{1}$ having an earlier EF time coordinate than $E_{2}$ ), whether they are causally related or not. To do this we perform the following algorithm:

1. Is $E_{1}$ is behind the horizon and $r_{2}>r_{1}$ ? If so they are unrelated.

2. Change the angular coordinates so that both lie on the equatorial plane, and restrict attention to null geodesics which traverse an azimuthal angle $\leq \pi$ on their trip from $E_{1}$ to a stationary worldline $\gamma$ containing $E_{2}$.

3. Is the EF time separation of the events less than the angular or radial spacelike bounds? If so they are unrelated.

\footnotetext{
${ }^{6}$ We chose $r_{\max }=3$ to get roughly the same number of interior and exterior events, and $t_{\max }=8$ to be roughly half the circumference of a circle at $r=3$. Note that $r=3$ corresponds to the innermost circular orbit (which is therefore lightlike).
} 
4. Is the EF time separation greater than the timelike bound? If so they are related.

5. If neither sufficient condition is satisfied, then we must numerically compute the value of $(E / L)^{2}$ which will send a null geodesic from $E_{1}$ to $\gamma$. Armed with this value, we compute the elapsed coordinate time along this geodesic, and decide if it arrives before or after the event $E_{2}$.

Table 2 gives details for a selection of pairs of events from Table 1, In particular we show the various quantities which are computed along the way to deciding if this pair is causally related. For each pair, if

\begin{tabular}{|c|c|c|c|c|c|c|c|c|c|c|}
\hline pair & dir & $r_{0}$ & $\varphi_{2}$ & $\overline{\Delta t}$ & rad trip & ang bnd & tot trip & $(E / L)^{2}$ & time & result \\
\hline 01 & out & 2.898906 & 2.567258 & 0.698520 & 4.179694 & 13.36484 & - & - & - & unrelated : either bound \\
\hline 02 & in & - & 0.475629 & 0.722210 & 1.000779 & 0.647253 & - & - & - & nd \\
\hline 12 & in & - & 2.937662 & 0.023690 & 1.538071 & 3.997673 & - & - & - & bound \\
\hline 04 & in & 2.361614 & 1.827161 & 2.825075 & 1.706999 & 1.196088 & 12.73426 & 0.0460462 & 4.69799 & lated \\
\hline 14 & in & 2.898906 & 0.965528 & 2.126556 & 2.244290 & 0.632050 & - & - & - & unrelated : radial bound \\
\hline 24 & in & 1.360835 & 1.97 & 2.102865 & 0.706219 & 1.2 & - & - & - & \\
\hline 05 & in & 2.361614 & 0.867289 & 3.561976 & 1.398076 & 0.835666 & 6.632326 & 0.272754 & 2.11915 & ted \\
\hline 15 & in & 2.898906 & 2.821962 & 2.863456 & 1.935368 & 2.719068 & 16.62616 & 0.0388018 & 9.86353 & elated \\
\hline 25 & in & 1.360835 & 0.512295 & 2.839766 & 0.397297 & 0.493616 & - & - & - & generic, hits singularity \\
\hline 45 & out & & & & & & & & \multicolumn{2}{|c|}{ emerging from interior : unrelated } \\
\hline 06 & in & 2.3616 & 2.8 & 2 & & 6.6 & - & - & - & unrelated : \\
\hline 16 & in & 2.898906 & 0.690536 & 4.121342 & 0.554150 & 1.619139 & 4.148999 & 0.0476468 & 2.60973 & generic, exterior, related \\
\hline 26 & out & & & & & & & & \multirow{3}{*}{\multicolumn{2}{|c|}{$\begin{array}{l}\text { emerging from interior : unrelated } \\
\text { emerging from interior : unrelated } \\
\text { emerging from interior : unrelated }\end{array}$}} \\
\hline 46 & out & & & & & & & & & \\
\hline 56 & out & & & & & & & & & \\
\hline 37 & in & 2.740935 & 0.480906 & 3.2708 & 2.0761 & 0.31 & 611431 & 4.55373 & 2.1833 & \\
\hline 58 & in & 0.963538 & 0.485959 & 2.220218 & 0.533902 & 0.208785 & - & 1.35646 & 0.667059 & generic, interior, related \\
\hline
\end{tabular}

Table 2: Considering relations between a collection of pairs of elements of Table1.

applicable, we show:

- the direction (ingoing or outgoing)

- the angle between the events $\varphi_{2}$

- the time coordinate separation between the events $\Delta t$

- the 'radial' spacelike bounds, given by Eq. (6) for ingoing null geodesics, and Eq. (7) for outgoing geodesics

- the 'angular' spacelike bound, Eq. (9)

- the coordinate time traversed along the trip, composed of successive segments at constant $r$ or angular position, which yields the timelike bound of Subsection 2.3.2

- the value of $c^{2}=(E / L)^{2}$ in Eq. (13) which yields the fastest null geodesic from $E_{1}$ to $\gamma$. These values are computed using the procedure detailed in Appendix B.2.

- the coordinate time elapsed along this fastest geodesic

- whether they are related or not, and which condition allows us to decide

For the 01 pair, the time coordinate separation $\Delta t$ is less than either of the spacelike bounds, so the events are unrelated. The 02 pair fails the angular spacelike bound, but passes the radial bound. The 04 pair is 'generic', meaning that it fails both spacelike bounds and the timelike bounds. These simple tricks are insufficient to 
determine if the events are related, so we must integrate Eq. (13) to locate the fastest null geodesic from event 0 to the $\gamma$ containing event 4. This geodesic crosses the horizon, but does not arrive at $\gamma$ in time for the events to be related (since the 'time' in the last column is larger than the 'available time' $\Delta t$ ). The 24 pair fails the radial spacelike bound. The radial and timelike bounds fail because the pair is inside the horizon, for which there is no timelike, constant- $r$ trajectory. Integrating (13), with $c^{2} \equiv(E / L)^{2}=0$, gives only $\varphi=0.721811$, which is not enough to reach $\gamma$. All null geodesics will hit the singularity before reaching the $\theta_{2}=0.11664, \phi_{2}=0.11664$ worldline. The 25 pair meets a similar fate: there are no future directed null geodesics from event 2 which reach $\theta_{2}=2.33727, \phi_{2}=1.38169$ before falling into the singularity. The 05 pair fails all bounds, and thus is generic. This time, however, the null geodesic does reach $\gamma$ before event 5 . The 45 pair represents an attempt to 'escape from the interior', in the sense that event 4 is inside the horizon, and event 5 is at a larger radius than 4. Even though event 5 is also inside the horizon, there are no causal curves inside the horizon which extend to larger radii. Events 0 and 6 are at almost the same radius, but at very different angular positions. Thus the angular bound is useful in deducing that they are unrelated, without having to integrate any geodesics. Events 1 and 6 are an example of a generic pair which are both outside of the horizon. They happen to be related. The pairs 26,46 , and 56 suffer the same fate as 45 . This time they are even attempting to escape across the horizon. The 58 pair is generic and completely inside the horizon. This time there are causal curves which reach $\gamma$ from event 5 , and the events end up being related.

\subsection{Causal Sets}

In this subsection we show some Hasse diagrams of the causal sets which arise from sprinkling into Schwarzschild. In the Hasse diagram one shows only the links of the causal set, namely those causal relations which are not implied by transitivity. Figures 3 and 4 use the graphviz package [17] to generate the diagram, ignoring the embedding information. Figures 5, 6, 7, and 8 arrange the causal set elements using their embedded location.

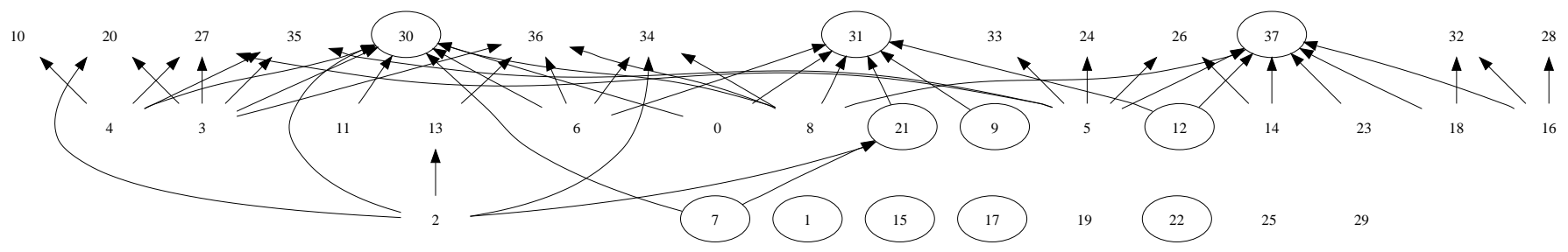

Figure 3: Hasse diagram of a 38 element causal set sprinkled into Schwarzschild spacetime, with $r_{\max }=$ $4, t_{\max }=8$. The circled elements landed behind the horizon. Note that no information escapes from this interior region, in that there are no causal relations from circled to uncircled elements.

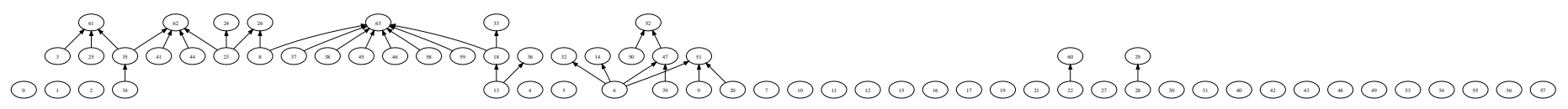

Figure 4: A 'causal set singularity'. This 64 element causet is sprinkled near the singularity of Schwarzschild, $r_{\max }=1, t_{\max } \rightarrow \infty$. (The resulting causet is independent of $t_{\max }$ beyond some finite value.)

Figure 3 shows a 38 element causal set, which arises from a sprinkling into a region of a Schwarzschild spacetime. Figure 4 portrays a causal set which arises when sprinkling near the singularity. Note that there is no need to worry about sprinkling on top of the singularity, as that is a zero probability event, even if the singularity is contained within the sprinkling region. It is very antichain-like, as the futures of the elements rapidly fall into the singularity, so it is unlikely that another sprinkled element lands in that region.

In the remaining four figures of this section we show more Hasse diagrams, though this time we use the embedding information to locate the nodes in the graph. For the first three, Figures 5, 6, and 7 we generate a causal set $C$ by sprinkling into half of the equatorial plane $(\theta=\pi / 2, \phi \in[0, \pi])$, with $t_{\max }=8$ and $r_{\max }=4$. The 


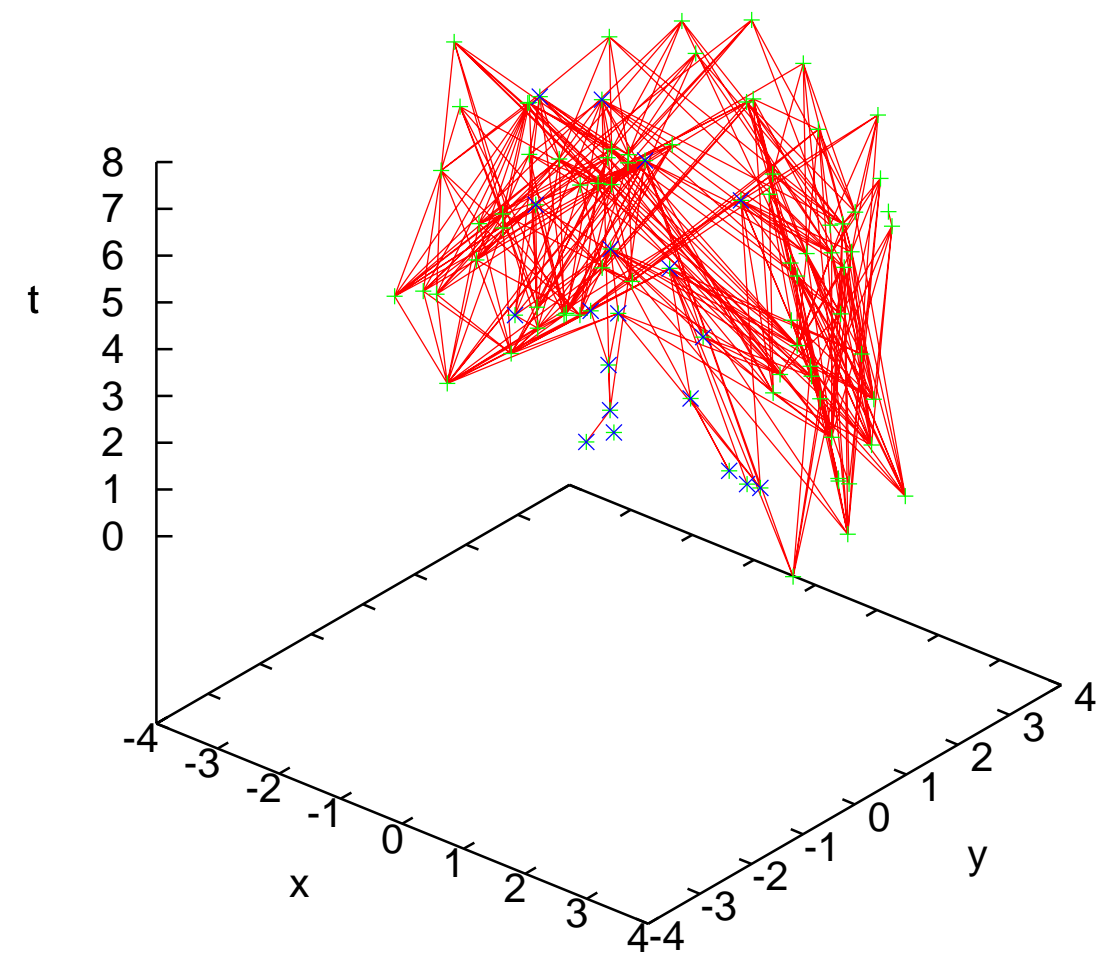

Figure 5: Hasse diagram of a causal set $C$ which arises from sprinkling 91 elements into the half equatorial plane. Elements sprinkled behind the horizon appear blue, while those outside are green. 


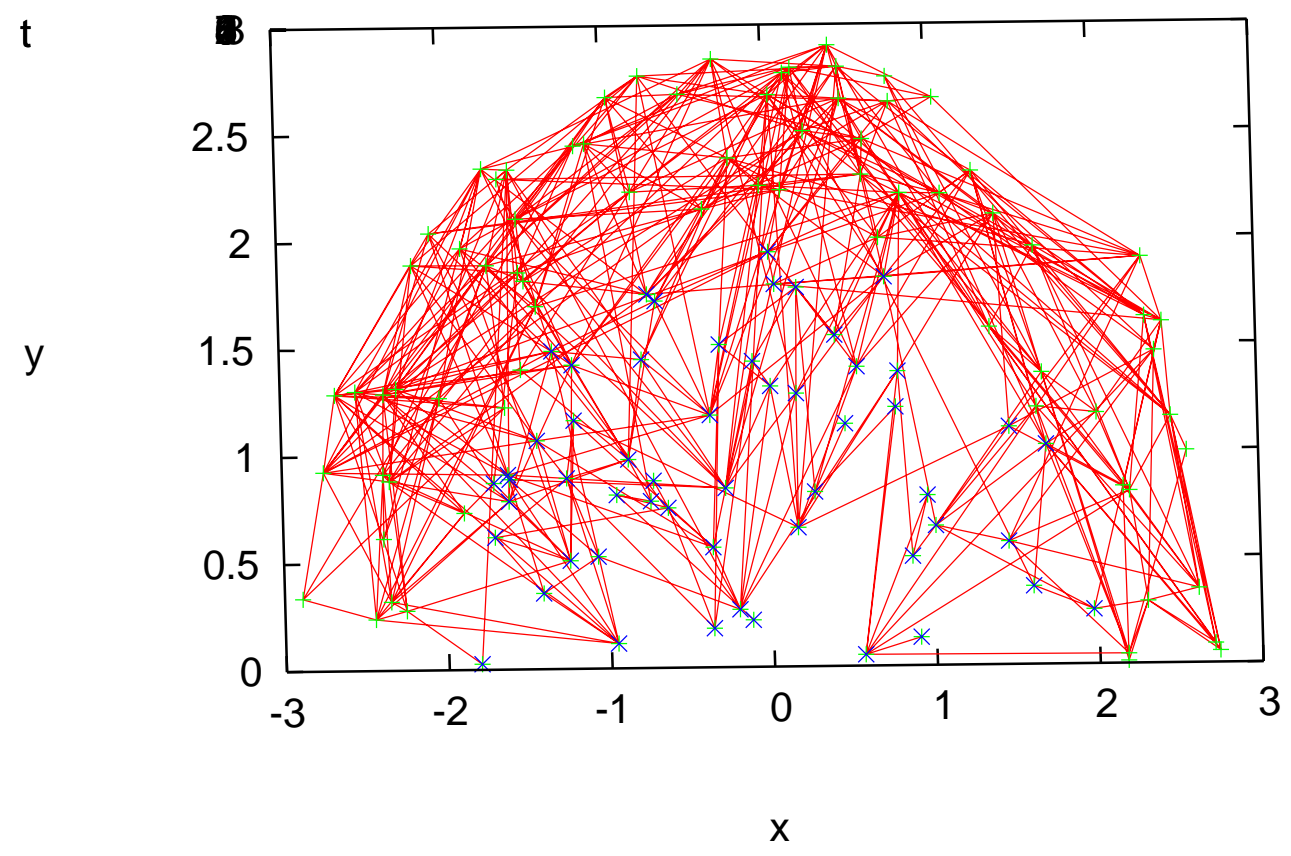

Figure 6: The same 91 element causal set $C$ as viewed from the 'top' ( $t \rightarrow+\infty$, look into the past direction). The blue 'half disk' corresponds to the black hole interior. 


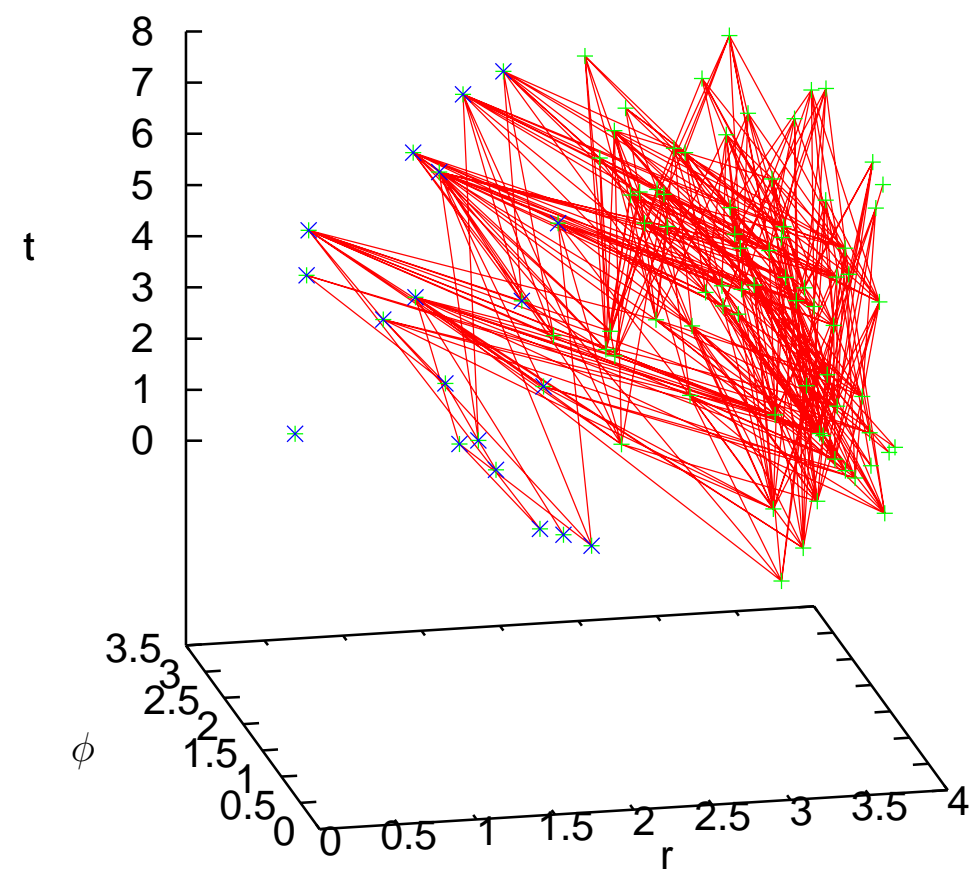

Figure 7: The sprinkled 91 element causal set $C$, this time plotting $r$ against $\phi$ interpreted as Cartesian coordinates, rather than polar coordinates. The blue region on the left corresponds to the interior of the black hole. 
relations (links) are shown in red. The half plane is chosen to reduce the 'clutter' from links between distant elements (such as links which cross the black hole, from the exterior region on one side to the exterior region on the other). Note that these red lines are not null geodesics connecting the elements (though they will converge to such in the infinite sprinkling density limit). They are simply straight lines between the elements drawn by the plotting program (gnuplot).

The last figure 8 attempts to more clearly show the light cone structure of Schwarzschild, in particular the tilting of the light cones toward the singularity at $r=0$. Here we sprinkle 91 elements into a 1/10 radian 'wedge' of the equatorial plane. Since the number of elements sprinkled into a region grows quadratically with $r$, we plot here $t$ versus $r^{2}$, so the distribution will appear approximately uniform in the horizontal direction. On the right side of the figure we have a cutoff at $r=4$, so obviously no links can go beyond that cutoff. However around $r=3$ we can see the light cone spreading in both directions. As we get closer to the center the links extend less and less to the right (exterior). As we cross the horizon at $r=2$ we see that the links only extend to the left, since their are no future directed causal curves which have non-decreasing $r$ in the interior.

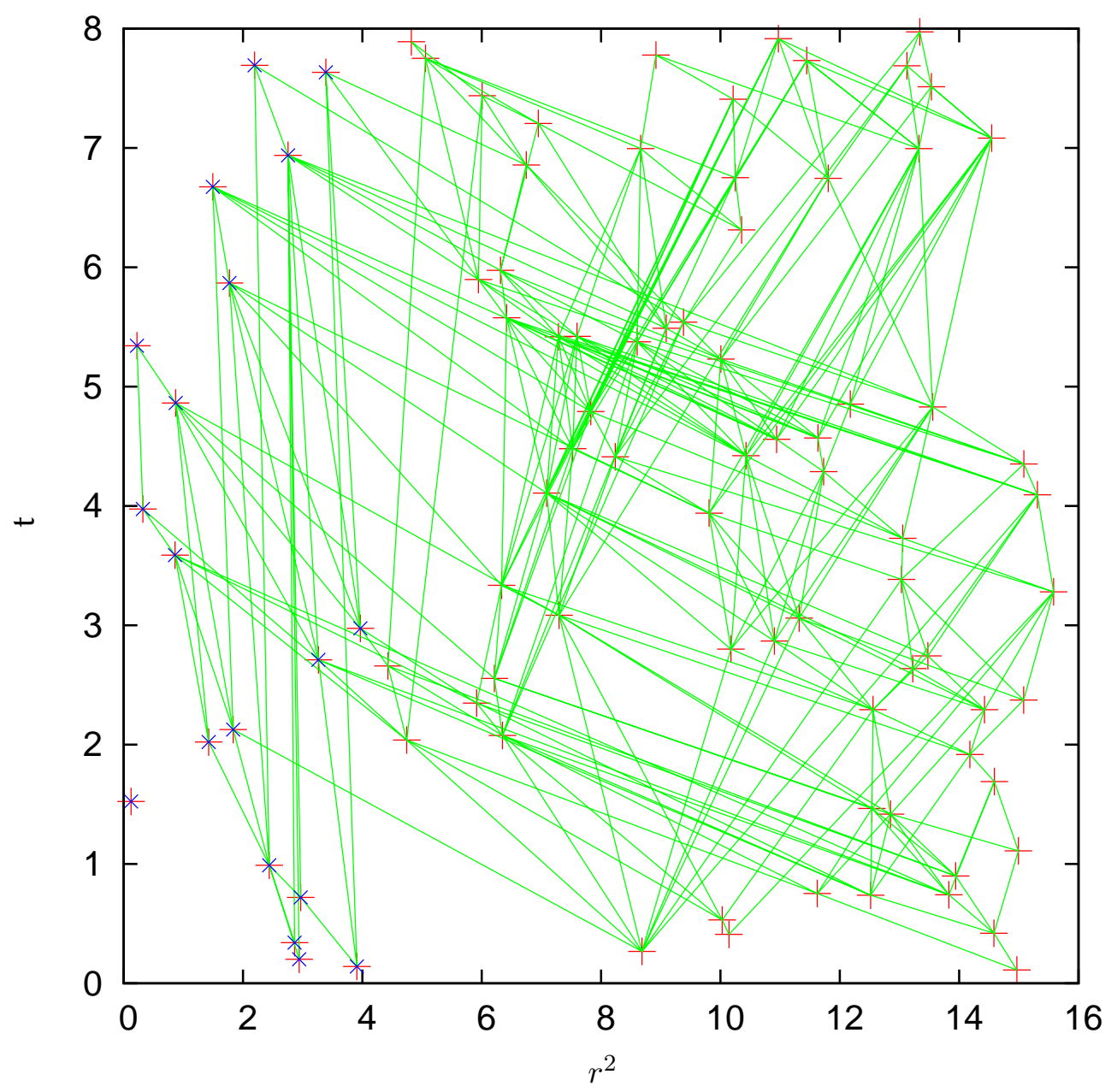

Figure 8: Sprinkling of 91 elements into a 1/10 radian wedge of the equatorial plane. Here we ignore the $\phi$ coordinate, and square the radial coordinate in order to spread out the elements at large radius. 


\section{Conclusion and discussion}

We have described an algorithm to determine if two events are causally related in Schwarzschild spacetime. It involves first checking a number of sufficient conditions, to see if these are able to determine whether they are related, without resorting to a time consuming numerical integration. If none of these are satisfied then we locate a future directed null geodesic which leads from the earlier event to a worldline containing the later, and integrate the elapsed EF time along the geodesic to determine if the pair is related.

We then wrote a 'thorn' (module) within the Cactus framework with sprinkles events into a region of Schwarzschild, and use this algorithm to deduce the causal relations between every pair of events. This procedure yields a causal set which 'faithfully embeds' into a Schwarzschild black hole spacetime.

It should not be difficult to generalize this prescription to other black hole spacetimes, such as ReissnerNordstrom or Kerr. In order to describe the closed timelike curves in interior regions one may generalize the definition of the causal set slightly, replacing the irreflexive condition with reflexivity, so one ends up with a transitive directed graph. In addition one can consider other conformally curved spacetimes, such as the $k= \pm 1$ Friedman-Robertson-Walker universes.

This work is a good starting point for one to address a wide array of questions within the causal set program, which previously were inaccessible. On one hand it will allow one to easily investigate many kinematical questions with regard to the so-called 'Hauptvermutung' of causal sets, which conjectures that if one has a causal set which is likely to arise from sprinkling into two separate spacetimes, then those spacetimes must be approximately isometric. To date, all results regarding to how to deduce properties of an approximating continuum from a causal set consider only conformally flat geometries. Now one will be able to test such constructs on a much wider class of geometries.

Another important application is towards our understanding of black hole thermodynamics. The fundamental discreteness of causal sets gives us a possible way to characterize the degrees of freedom which give rise to black hole entropy. We are now in a position to repeat the analysis of the link counting and its generalizations [12], for the full $4 \mathrm{~d}$ Schwarzschild geometry. As argued by Dou and Sorkin, this may provide access to the fundamental length scale of quantum gravity, since entropy, as a pure number, is not subject to renormalization.

Finally, a long standing question in semi-classical gravity is the trans-Planckian problem, that the Hawking radiation emanating from a black hole horizon, at late times (after the black hole forms), arises from modes of frequency much greater than the Planck scale [18]. If spacetime is discrete at this scale, then one may expect that such trans-Planckian modes cannot exist. How then is Hawking radiation possible, in such a discrete setting? Important groundwork on the dynamics of scalar fields on a background causal set has recently been laid [19, 20, 21]. Now that we can construct causal sets which correspond to a full four dimensional black hole, it may be possible to address this question within the causal set approach.

\section{Acknowledgments}

We are extremely grateful to Joseph Samuel and Rafael Sorkin for illuminating discussions on the behavior of null geodesics and their relation to causal structure. SH also thanks Hongbao Zhang for many helpful discussions and comments.

This research was supported by the Perimeter Institute for Theoretical Physics. Research at Perimeter Institute is supported by the Government of Canada through Industry Canada and by the Province of Ontario through the Ministry of Research \& Innovation. SH was supported by NFSC grants 10235040 and 10421003.

$\mathrm{SH}$ thanks the Perimeter Institute for hospitality while this work was carried out. 


\section{A Appendix: Proof of the proposition}

In this section, we want to prove the following proposition. Given two events $E_{1}$ and $E_{2}$ in Schwarzschild spacetime, with $t_{1}<t_{2}$, and $E_{2}$ representing the moment $t_{2}$ on the world line $\gamma$ of a stationary observer, the null geodesic with $|\Delta \varphi|=\varphi_{2}$ is the fastest one (arrives at the earliest time in $E F$ coordinates) for all futuredirected null geodesics from $E_{1}$ to $\gamma$.

It is clear that the fastest geodesic will have the least elapsed time. Thus from Eq. (15), we wish to minimize the integral

$$
\Delta t=\int_{u_{1}}^{u_{2}} \frac{ \pm c\left(2 M u^{3}-u^{2}+c^{2}\right)^{-1 / 2}-2 M u}{u^{2}-2 M u^{3}} d u
$$

along the geodesic. Since $d \varphi$ is clearly nonnegative, the sign in front of $c$ will be positive for ingoing geodesics $(d u>0)$ and negative for outgoing geodesics $(d u<0)$. Given $E_{1}$ and $E_{2}$ (and thus $\gamma$ ), different null geodesics are given by different values of $c$, and it is straightforward to see from Eq. (16) that in every case for larger $c$, $\Delta t$ gets smaller. Therefore, the fastest null geodesic from $E_{1}$ to $\gamma$ must have the largest possible $c$ to reach $\gamma$, which, by Eq. (13), has the smallest possible $|\Delta \varphi|=\varphi_{2} \square$

\section{B Numerical Details}

\section{B.1 Sprinkling into Schwarzschild Spacetime}

In implementing these ideas on a computer, the first task is to randomly select events in spacetime, with a density proportional to the spacetime volume factor

$$
\sqrt{-g}=r \sqrt{r^{2}+12 M^{2}} \sin \theta
$$

Because of the simple product form of this expression, we can break up the sprinkling into an angular piece, a temporal piece, and a radial piece. For the uniform sprinkling of the angular coordinates, on a 2-sphere, we follow the procedure described in Section 5.2 of [22]. For the temporal sprinkling, since the volume element is independent of $t$, we can select its values uniformly at random.

The sprinkling in the radial direction must be performed such that it yields the distribution of Eq. (17) (ignoring the $\theta$ dependence; this is accounted for above). This is achieved by the following general method (derived from [23]). To sprinkle a coordinate $x$ between the bounds $a \leq x \leq b$ such that it has distribution $f(x)$, compute the indefinite integral

$$
I(x)=\frac{1}{N} \int_{a}^{x} f\left(x^{\prime}\right) d x^{\prime},
$$

where the constant normalization factor is

$$
N=\int_{a}^{b} f\left(x^{\prime}\right) d x^{\prime}
$$

Now invert Eq. (18) to get $x$ as a function of $I$. This expression, where $I$ is a random variable distributed uniformly in the unit interval [0,1], will be distributed according to $f(x)$. Of course this method only works if $f(x)$ is integrable and its integral is invertible. In our case $f(r)=r \sqrt{r^{2}+12 M^{2}}$, and the expression is $\sqrt{\left(3 N I+\left(a^{2}+12 M^{2}\right)^{3 / 2}\right)^{2 / 3}-12 M^{2}} \cdot 8$

\footnotetext{
${ }^{7}$ As mentioned earlier, for $\varphi_{2}<\pi$, there is only one fastest null geodesic with the largest $c$ to make $\Delta \varphi=\varphi_{2}$; while for $\varphi_{2}=\pi$, there are in fact two fastest null geodesics with $\Delta \varphi= \pm \pi$ but the same $c$ and $\Delta t$.

${ }^{8}$ In fact the angular sprinkling is of this type as well. With $f(\theta)=\sin \theta$ the expression is $\arccos (2 I-1)$. The $\phi \operatorname{coordinate}$ is distributed uniformly in $[0,2 \pi]$.
} 


\section{B.2 Determining the Causal Relations}

Once equipped with a collection of events in Schwarzschild, we can sort them by their time coordinate, and then consider each sorted pair in turn. The first task is to check the sufficient conditions described in Subsection 2.3 . This is relatively straightforward; numerous examples are given in Subsection 3.1. If the pair fails all available sufficient conditions, then it is a 'generic pair', and we must integrate null geodesics as described in Subsection 2.4 .

The basic task is to find a value of the parameter $c^{2}=(E / L)^{2}$ for which the integral of Eq. (13) along the null geodesic from $u_{1}$ to $u_{2}$ equals $\varphi_{2}$. This is made complicated by the fact that the cubic $f(u)=2 M u^{3}-u^{2}+c^{2}$ in the denominator of the right hand side can have real roots within the domain of integration. (See Fig. 9 for an illustration.) The behavior of these roots is as follows. For $c^{2}>\frac{1}{27 M^{2}}$, the cubic has no non-negative real roots.

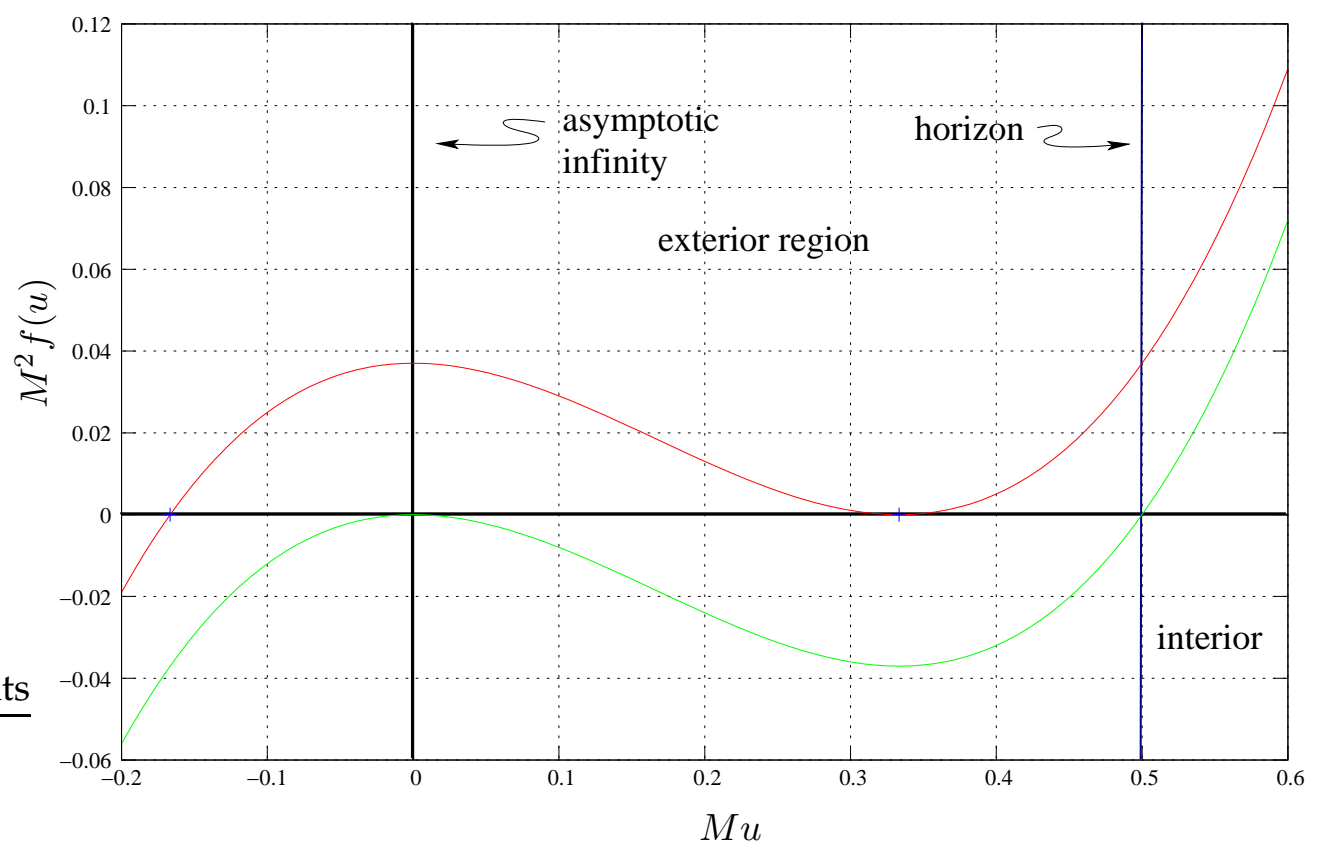

Figure 9: Cubic $f(u)=2 M u^{3}-u^{2}+c^{2}$ in denominator of Eq. 13). The red curve has $c^{2}=\frac{1}{27 M^{2}}$, while the green has $c^{2}=0$.

At $c^{2}=\frac{1}{27 M^{2}}$ it has a double root at $u=\frac{1}{3 M}$ (and a third at $u=\frac{-1}{6 M}$, which is irrelevant because we consider only non-negative values of $u=1 / r$ ). This double root, which causes the integrand to diverge, corresponds to the pure angular orbit at $u=\frac{1}{3 M}$. As we further reduce $c^{2}$, the double root at $u=\frac{1}{3 M}$ separates into two, the larger increasing toward $u=\frac{1}{2 M}$ (the horizon), and the smaller decreasing toward $u=0$ (asymptotic infinity), which they reach when $c^{2}$ descends to its smallest possible value 0 . So the task of our algorithm is to shoot null geodesics from $E_{1}$ in different directions (different values of $c^{2}$ ), trying to hit $\gamma\left(\varphi_{2}\right)$, all the time being careful to keep $c^{2}$ large enough that the roots of the cubic do not fall between either $u_{1}$ or $u_{2}$, as doing so would cause the integrand to become infinite or imaginary.

Our algorithm begins by setting $c^{2}=0$ if either event lines behind the horizon, or $c^{2}=\frac{1}{27 M^{2}}$ otherwise. It will later adjust the value of $c^{2}$, in its attempt to locate a null geodesic which travels from $E_{1}$ to $\gamma$. For each value of $c^{2}$, it first checks to see if either $u_{1}$ or $u_{2}$ lies between the non-negative real roots (if any). If so, it adjusts $c^{2}$ upward (by using a linear extrapolation of $f(u)$ at the roots), until neither $u_{1}$ or $u_{2}$ lies between them.

Now that we have an integrand which is real and finite in the entire domain $\left[u_{1}, u_{2}\right]$, the code integrates Eq. (13) numerically using the composite Simpson's rule, with $n=512$ subintervals. If either the numerically evaluated integral is within $\Delta=5 \epsilon$ of $\varphi_{2}$, where $\epsilon$ is double precision machine epsilon $\left(\approx 2.22045 \times 10^{-16}\right.$ on the machine on which the results of section 3 were generated), or the two most recently chosen values of $c^{2}$ are within $5 \epsilon$ of each other (see below), we then (tentatively) decide that this null geodesic arrives at $\gamma$. If this condition fails, we choose a second value for $c^{2}$ and repeat. The second value is chosen to be the the first +.03 
if the integral overshoots (is greater than) $\varphi_{2}$, or the first - .005 if it undershoots. If the second guess of $c^{2}$ also misses $\varphi_{2}$, then subsequent values are chosen by a linear interpolation/extrapolation from the two previous guesses. This algorithm (with the additional features described below) converges for all pairs of events we have encountered in our simulations.

There is a special situation which can arise (as in some of the examples of Subsection 3.1), in which there are no causal curves from $E_{1}$ to $\gamma$. This occurs when $E_{2}$ lies behind the horizon, and the integral with $c^{2}=0$ undershoots $\varphi_{2}$. This means that every future directed causal curve from $E_{1}$ falls into the singularity before reaching $\gamma$, so the events must be unrelated.

When $c^{2}$ is small enough that the domain of integration touches a root, the integral diverges. Often the 'target value' of $\varphi_{2}$ requires a $c^{2}$ which is is very close to this singular value. We find that a convenient way to handle this situation numerically is to detect when we manage to find a valid value of $c^{2}$, which is large enough for $u_{1}$ and $u_{2}$ to escape the roots, and yet small enough to yield an integral which exceeds $\varphi_{2}$. Once we find this value of $c^{2}=c_{\min }^{2}$, then we know that the value of $c^{2}$ we seek is greater than this. Thus, in the course of the above iteration, which uses linear interpolation/extrapolation to select subsequent values of $c^{2}$, if a value is selected which is smaller than $c^{2}=c_{\min }^{2}$, then we instead choose the mean of $c^{2}=c_{\min }^{2}$ and the previous $c^{2}$. (Furthermore in subsequent iterations, if we find a yet larger value of $c^{2}$ for which the integral exceeds $\varphi_{2}$, then we use this as the new $c_{\text {min }}^{2}$.)

Once the above loop converges, so that we have a value of $c^{2}$ for which the integral of Eq. (13) yields $\varphi_{2}$, we then check that the numerical approximation to the integral is sufficiently accurate. The check is simple: we compute the numerical approximation to the integral again at four times the resolution $\varphi_{2}(4 n)(4 n$ subintervals), and subtract that value from the $\varphi_{2}(n)$ using $n$ subintervals. If the difference is greater than $8 \eta$, where $\eta$ is the larger of $\varphi_{2}(4 n)-\varphi_{2}$ and $5 \epsilon$, then we double $n$ and repeat the above iteration. (Though we stop the iteration if the difference $\left|\varphi_{2}(4 n)-\varphi_{2}(n)\right|$ ever increases from that for the previous $n$.)

Now that we have an accurate value of $c^{2}$, which yields a null geodesic which hits $\gamma$, we integrate Eq. (15) to get the elapsed time along the geodesic, and thus can determine if the two events are related by comparing this elapsed time with $t_{2}-t_{1}$.

\section{References}

[1] S. Chandrasekhar, Mathematical Theory of Black Holes, Oxford University Press, 1998.

[2] R.J. Low, "The Space of Null Geodesics (and a New Causal Boundary)" in Lecture Notes in Physics 692 pp. 35-50, Springer Berlin / Heidelberg (2006).

[3] R.J. Low, "Twistor linking and causal relations in exterior Schwarzschild space", Class. Quant. Grav. 11 pp. 453-456 (1994).

[4] L. Bombelli, J.H. Lee, D. Meyer, and R. Sorkin, “Space-time as a causal set," Phys. Rev. Lett. 59 (1987) 521.

[5] Jan Myrheim, “Statistical Geometry,” CERN preprint Ref.TH.2538-CERN (1978).

[6] G. Brightwell and R. Gregory, "The Structure of random discrete space-time," Phys. Rev. Lett. 66, 260 (1991).

E. Bachmat, "Discrete spacetime and its applications," 〈e-print arXiv: gr-qc/0702140〉.

[7] R. D. Sorkin, "Space-time and causal sets," in Relativity and Gravitation: Classical and Quantum (Proceedings of the SILARG VII Conference, Cocoyoc, Mexico, December 1990), pp. 150-173. World Scientific, Singapore, 1991.

M. Ahmed, S. Dodelson, P. B. Greene and R. Sorkin, “Everpresent Lambda,” Phys. Rev. D 69, 103523 (2004) 〈e-print arXiv: astro-ph/0209274〉. 
[8] D.A. Meyer, "Spherical containment and the Minkowski dimension of partial orders," Order 10 227-237 (1993).

— The Dimension of Causal Sets, PhD Thesis, M.I.T. (1988).

D. D. Reid, “The manifold dimension of a causal set: Tests in conformally flat space-times," Phys. Rev. D 67, 024034 (2003).

[9] R. Ilie, G. B. Thompson and D. D. Reid, "A numerical study of the correspondence between paths in a causal set and geodesics in the continuum," Class. Quant. Grav. 23, 3275 (2006).

[10] D. Rideout and P. Wallden, "Spacelike distance from discrete causal order," 〈e-print arXiv: 0810.1768 [gr$\mathrm{qc}]\rangle$.

[11] S. Major, D. Rideout, and S. Surya, “On recovering continuum topology from a causal set," J. Math. Phys. 48032501 (2007). 〈e-print arXiv: gr-qc/0009063〉.

S. Major, D. Rideout, and S. Surya, "Stable Homology as an Indicator for Manifoldlikeness in Causal Sets", in preparation.

[12] D. Dou and R. D. Sorkin, "Black Hole Entropy as Causal Links," Found. Phys. 33, 279 (2003). (e-print arXiv: gr-qc/0302009〉.

S. Marr, Black hole entropy from Causal Sets, PhD Thesis, Imperial College London (2007).

D. Rideout and S. Zohren, "Counting entropy in causal set quantum gravity," in Proceedings of the Eleventh Marcel Grossmann Meeting on General Relativity, (ed.) H. Kleinert, R.T. Jantzen and R. Ruffini, World Scientific, (2008), p. 2803. 〈e-print arXiv: gr-qc/0612074〉.

[13] D. Rideout and S. Zohren, "Evidence for an entropy bound from fundamentally discrete gravity," Class. Quant. Grav.23 6195 (2006). 〈e-print arXiv: gr-qc/0606065〉.

[14] T. Goodale, G. Allen, G. Lanfermann, J. Massó, T. Radke, E. Seidel, and J. Shalf, “The Cactus Framework and Toolkit: Design and Applications" in Vector and Parallel Processing - VECPAR 2002, 5th International Conference, Springer, pp. 197-227.

[15] R. Penrose, Techniques of Differential Topology in Relativity, Society for Industrial and Applied Mathematics, 1972.

[16] See for example p. 167 of E. Poisson, A Relativist's Toolkit:The Mathematics of Black-hole Mechanics, Cambridge University Press, 2004.

[17] www.graphviz.org

[18] T. Jacobson, "Introduction to Quantum Fields in Curved Spacetime and the Hawking Effect," in Valdivia 2002, Lectures on Quantum Gravity, pp. 39-89 (2005).

[19] R. D. Sorkin, "Does locality fail at intermediate length-scales?", to appear in Towards Quantum Gravity, D. Oriti (ed.), Cambridge University Press. 〈e-print arXiv: gr-qc/0703099〉.

J. Henson, "The causal set approach to quantum gravity," in Approaches to Quantum Gravity - Towards a new understanding of space and time, D. Oriti, ed. Cambridge University Press, 2006. 〈e-print arXiv: grqc/0601121).

[20] Steven Johnston, "Particle propagators on discrete spacetime," Class. Quant. Grav.25 202001 (2008). 〈e-print arXiv: 0806.3083 [hep-th] $\rangle$.

[21] Roman Sverdlov and Luca Bombelli, "Gravity and Matter in Causal Set Theory," (2008). 〈e-print arXiv: 0801.0240 [gr-qc] $\rangle$. 
[22] J. Brunnemann and D. Rideout, "Properties of the Volume Operator in Loop Quantum Gravity II: Detailed Presentation," Class. Quant. Grav.25 065002 (2008). 〈e-print arXiv: 0706.0382 [gr-qc]〉.

[23] Donald E. Knuth, The Art of Computer Programming, Volume 2: Seminumerical Algorithms, Third Edition, Addison-Wesley (1998). 